ELECTRONIC RESEARCH ANNOUNCEMENTS OF THE AMERICAN MATHEMATICAL SOCIETY

Volume 7, Pages 54-62 (June 26, 2001)

S $1079-6762(01) 00094-4$

\title{
GENERATORS AND RELATIONS FOR SCHUR ALGEBRAS
}

\author{
STEPHEN DOTY AND ANTHONY GIAQUINTO
}

(Communicated by Alexandre Kirillov)

\begin{abstract}
We obtain a presentation of Schur algebras (and $q$-Schur algebras) by generators and relations, one which is compatible with the usual presentation of the enveloping algebra (quantized enveloping algebra) corresponding to the Lie algebra $\mathfrak{g l}_{n}$ of $n \times n$ matrices. We also find several new bases of Schur algebras.
\end{abstract}

\section{INTRODUCTION}

The classical Schur algebra $S(n, d)$ (over $\mathbb{Q}$ ) may be defined as the algebra $\operatorname{End}_{\Sigma_{d}}\left(V^{\otimes d}\right)$ of linear endomorphisms on the $d$ th tensor power of an $n$-dimensional $\mathbb{Q}$-vector space $V$ commuting with the action of the symmetric group $\Sigma_{d}$, acting by permutation of the tensor places (see [6]). Schur algebras determine the polynomial representation theory of general linear groups, and they form an important class of quasi-hereditary algebras.

We identify $V$ with $\mathbb{Q}^{n}$. Then $V_{\mathbb{Z}}=\mathbb{Z}^{n}$ is a lattice in $V$. One can define a $\mathbb{Z}$-order $S_{\mathbb{Z}}(n, d)$ (the integral Schur algebra) in $S(n, d)$ by setting $S_{\mathbb{Z}}(n, d)=\operatorname{End}_{\Sigma_{d}}\left(V_{\mathbb{Z}}^{\otimes d}\right)$. For any field $K$, one then obtains the Schur algebra $S_{K}(n, d)$ over $K$ by setting $S_{K}(n, d)=S_{\mathbb{Z}}(n, d) \otimes_{\mathbb{Z}} K$. Moreover, $S_{\mathbb{Q}}(n, d) \cong S(n, d)$.

In the quantum case one can replace $\mathbb{Q}$ by $\mathbb{Q}(v)$ ( $v$ an indeterminate), $V$ by an $n$-dimensional $\mathbb{Q}(v)$-vector space, and $\Sigma_{d}$ by the corresponding Hecke algebra $\mathbf{H}\left(\Sigma_{d}\right)$. Then the resulting commuting algebra, $\mathbf{S}(n, d)$, is known as the $q$-Schur algebra, or quantum Schur algebra. It appeared first in work of Dipper and James, and, independently, Jimbo. Beilinson, Lusztig, and MacPherson [1] have given a geometric construction of $\mathbf{S}(n, d)$ in terms of orbits of flags in vector spaces. (See also [5].)

In this situation $\mathbb{Z}$ is replaced by the $\operatorname{ring} \mathcal{A}=\mathbb{Z}\left[v, v^{-1}\right]$, and there is a corresponding "integral" form $\mathbf{S}_{\mathcal{A}}(n, d)$ in $\mathbf{S}(n, d)$. The above quantized objects specialize to their classical versions when $v=1$. We have more detailed information about the Schur algebras in rank 1; see [2] and [3]. Proofs of the main results will appear in 4 .

Received by the editors April 8, 2001.

2000 Mathematics Subject Classification. Primary 16P10, 16S15; Secondary 17B35, 17 B37.

Key words and phrases. Schur algebras, finite-dimensional algebras, enveloping algebras, quantized enveloping algebras. 


\section{SERRE'S PRESENTATION OF $U$}

Let $\Phi=\left\{\varepsilon_{i}-\varepsilon_{j} \mid 1 \leq i \neq j \leq n\right\}$ be the root system of type $A_{n-1}$, with simple roots $\Delta=\left\{\varepsilon_{i}-\varepsilon_{i+1} \mid i=1, \ldots, n-1\right\}$, where $\left\{\varepsilon_{1}, \ldots, \varepsilon_{n}\right\}$ denotes the standard basis of $\mathbb{Z}^{n}$. The corresponding set $\Phi^{+}$of positive roots is the set $\Phi^{+}=\left\{\varepsilon_{i}-\varepsilon_{j} \mid\right.$ $1 \leq i<j \leq n\}$.

The abelian group $\mathbb{Z}^{n}$ has a bilinear form $\left(\right.$, ) given by $\left(\varepsilon_{i}, \varepsilon_{j}\right)=\delta_{i j}$ (Kronecker delta). We write $\alpha_{j}:=\varepsilon_{j}-\varepsilon_{j+1}$.

The enveloping algebra $U=U\left(\mathfrak{g l}_{n}\right)$ is the associative algebra (with 1) on generators $e_{i}, f_{i}(i=1, \ldots, n-1)$ and $H_{i}(i=1, \ldots, n)$ with relations

$$
\begin{gathered}
H_{i} H_{j}=H_{j} H_{i}, \\
e_{i} f_{j}-f_{j} e_{i}=\delta_{i j}\left(H_{j}-H_{j+1}\right), \\
H_{i} e_{j}-e_{j} H_{i}=\left(\varepsilon_{i}, \alpha_{j}\right) e_{j}, \quad H_{i} f_{j}-f_{j} H_{i}=-\left(\varepsilon_{i}, \alpha_{j}\right) f_{j}, \\
e_{i}^{2} e_{j}-2 e_{i} e_{j} e_{i}+e_{j} e_{i}^{2}=0 \quad(|i-j|=1), \\
e_{i} e_{j}-e_{j} e_{i}=0 \quad(\text { otherwise }), \\
f_{i}^{2} f_{j}-2 f_{i} f_{j} f_{i}+f_{j} f_{i}^{2}=0 \quad(|i-j|=1), \\
f_{i} f_{j}-f_{j} f_{i}=0 \quad \text { (otherwise). }
\end{gathered}
$$

For $\alpha \in \Phi$, let $x_{\alpha}$ denote the corresponding root vector of $\mathfrak{g l}_{n}$ viewed as an element of $U$. We have in particular $e_{i}=x_{\alpha_{i}}$ and $f_{i}=x_{-\alpha_{i}}$.

\section{The Quantized enVeloping Algebra}

The Drinfeld-Jimbo quantized enveloping algebra $\mathbf{U}=\mathbf{U}_{v}\left(\mathfrak{g l}_{n}\right)$, by definition, is the $\mathbb{Q}(v)$-algebra with generators $E_{i}, F_{i}(1 \leq i \leq n-1), K_{i}^{ \pm 1}(1 \leq i \leq n)$ and relations

$$
\begin{aligned}
& K_{i} K_{j}=K_{j} K_{i}, \quad K_{i} K_{i}^{-1}=K_{i}^{-1} K_{i}=1, \\
& E_{i} F_{j}-F_{j} E_{i}=\delta_{i j} \frac{K_{i} K_{i+1}^{-1}-K_{i}^{-1} K_{i+1}}{v-v^{-1}}, \\
& K_{i} E_{j}=v^{\left(\varepsilon_{i}, \alpha_{j}\right)} E_{j} K_{i}, \quad K_{i} F_{j}=v^{-\left(\varepsilon_{i}, \alpha_{j}\right)} F_{j} K_{i}, \\
& E_{i}^{2} E_{j}-\left(v+v^{-1}\right) E_{i} E_{j} E_{i}+E_{j} E_{i}^{2}=0 \quad(|i-j|=1), \\
& E_{i} E_{j}-E_{i} E_{j}=0 \quad(\text { otherwise }), \\
& F_{i}^{2} F_{j}-\left(v+v^{-1}\right) F_{i} F_{j} F_{i}+F_{j} F_{i}^{2}=0 \quad(|i-j|=1), \\
& F_{i} F_{j}-F_{j} F_{i}=0 \quad \text { (otherwise). }
\end{aligned}
$$

For $\alpha \in \Phi^{+}$, let $E_{\alpha}$ and $F_{\alpha}$ be the positive and negative quantum root vectors of $\mathbf{U}$ as defined by Jimbo [8]. We have in particular $E_{i}=E_{\alpha_{i}}$ and $F_{i}=F_{\alpha_{i}}$.

\section{Main Results: Classical CASE}

We now give a precise statement of our main results in the classical case. The first result describes a presentation by generators and relations of the Schur algebra over the rational field $\mathbb{Q}$. This presentation is compatible with the usual presentation (see section 2) of $U=U\left(\mathfrak{g l}_{n}\right)$. 
Theorem 1. Over $\mathbb{Q}$, the Schur algebra $S(n, d)$ is isomorphic to the associative algebra (with 1) on the same generators as for $U$ subject to the same relations (R1) - ( $\mathrm{R} 5$ as for $U$, together with the additional relations:

$$
\begin{aligned}
& H_{1}+H_{2}+\cdots+H_{n}=d, \\
& H_{k}\left(H_{k}-1\right) \cdots\left(H_{k}-d\right)=0 \quad(k=1, \ldots, n) .
\end{aligned}
$$

The next result gives a basis for the Schur algebra which is the analogue of the Poincare-Birkhoff-Witt (PBW) basis of $U$.

Theorem 2. The algebra $S(n, d)$ has a "truncated $P B W$ " basis (over $\mathbb{Q}$ ) which can be described as follows. Fix an integer $k_{0}$ with $1 \leq k_{0} \leq n$ and set

$$
G=\left\{x_{\alpha} \mid \alpha \in \Phi\right\} \cup\left\{H_{k} \mid k \in\{1, \ldots, n\}-\left\{k_{0}\right\}\right\}
$$

and fix some ordering for this set. Then the set of all monomials in $G$ (with specified order) of total degree at most $d$ is a basis for $S(n, d)$.

Our next result constructs the integral Schur algebra $S_{\mathbb{Z}}(n, d)$ in terms of the generators given above. We need some more notation. For $B$ in $\mathbb{N}^{n}$, we write

$$
H_{B}=\prod_{k=1}^{n}\left(\begin{array}{c}
H_{k} \\
b_{k}
\end{array}\right) \text {. }
$$

Let $\Lambda(n, d)$ be the subset of $\mathbb{N}^{n}$ consisting of those $\lambda \in \mathbb{N}^{n}$ satisfying $|\lambda|=d$; this is the set of $n$-part compositions of $d$. Given $\lambda \in \Lambda(n, d)$ we set $1_{\lambda}:=H_{\lambda}$. One can show that the collection $\left\{1_{\lambda}\right\}$ as $\lambda$ varies over $\Lambda(n, d)$ forms a set of pairwise orthogonal idempotents in $S_{\mathbb{Z}}(n, d)$ which sum to the identity element.

For $m \in \mathbb{N}$ and $\alpha \in \Phi$, set $x_{\alpha}^{(m)}:=x_{\alpha}^{m} /(m !)$. Any product in $U$ of elements of the form

$$
x_{\alpha}^{(m)}, \quad\left(\begin{array}{c}
H_{k} \\
m
\end{array}\right) \quad(m \in \mathbb{N}, \alpha \in \Phi, k \in\{1, \ldots, n\}),
$$

taken in any order, will be called a Kostant monomial. Note that the set of Kostant monomials is multiplicatively closed, and spans $U_{\mathbb{Z}}$. We define a function $\chi$ (content function) on Kostant monomials by setting

$$
\chi\left(x_{\alpha}^{(m)}\right):=m \varepsilon_{\max (i, j)}, \quad \chi\left(\left(\begin{array}{c}
H_{k} \\
m
\end{array}\right)\right):=0,
$$

where $\alpha=\varepsilon_{i}-\varepsilon_{j}(i \neq j)$, and by declaring that $\chi(X Y)=\chi(X)+\chi(Y)$ whenever $X, Y$ are Kostant monomials.

We write any $A \in \mathbb{N}^{\Phi^{+}}$in "multi-index" form $A=\left(a_{\alpha}\right)_{\alpha \in \Phi^{+}}$and set $|A|:=$ $\sum_{\alpha \in \Phi^{+}} a_{\alpha}$. For $A, C \in \mathbb{N}^{\Phi^{+}}$we write

$$
e_{A}=\prod_{\alpha \in \Phi^{+}} x_{\alpha}^{\left(a_{\alpha}\right)}, \quad f_{C}=\prod_{\alpha \in \Phi^{-}} x_{\alpha}^{\left(c_{\alpha}\right)}
$$

where the products in $e_{A}$ and $f_{C}$ are taken relative to any fixed orders on $\Phi^{+}$and $\Phi^{-}$.

Theorem 3. The integral Schur algebra $S_{\mathbb{Z}}(n, d)$ is the subring of $S(n, d)$ generated by all divided powers $e_{j}^{(m)}, f_{j}^{(m)}(j \in\{1, \ldots, n-1\}, m \in \mathbb{N})$. Moreover, this algebra has a $\mathbb{Z}$-basis consisting of all

$$
e_{A} 1_{\lambda} f_{C} \quad\left(\chi\left(e_{A} f_{C}\right) \preceq \lambda\right)
$$


and another such basis consisting of all

$$
f_{A} 1_{\lambda} e_{C} \quad\left(\chi\left(f_{A} e_{C}\right) \preceq \lambda\right)
$$

where $A, C \in \mathbb{N}^{\Phi^{+}}, \lambda \in \Lambda(n, d)$, and where $\preceq$ denotes the componentwise partial ordering on $\mathbb{N}^{n}$.

Finally, we have another presentation of the Schur algebra by generators and relations. This presentation has the advantage that it possesses a quantization of the same form, in which we can set $v=1$ to recover the classical version.

Theorem 4. For each $i \in\{1, \ldots, n-1\}$ write $\alpha_{i}:=\varepsilon_{i}-\varepsilon_{i+1}$. The algebra $S(n, d)$ is the associative algebra (with 1 ) given by generators $1_{\lambda}(\lambda \in \Lambda(n, d)), e_{i}, f_{i}$ $(i \in\{1, \ldots, n-1\})$ subject to the relations

$$
\begin{aligned}
& 1_{\lambda} 1_{\mu}=\delta_{\lambda, \mu} 1_{\lambda} \quad(\lambda, \mu \in \Lambda(n, d)), \\
& \sum_{\lambda \in \Lambda(n, d)} 1_{\lambda}=1 \\
& e_{i} 1_{\lambda}= \begin{cases}1_{\lambda+\alpha_{i}} e_{i} & \text { if } \lambda+\alpha_{i} \in \Lambda(n, d), \\
0 & \text { otherwise }\end{cases} \\
& f_{i} 1_{\lambda}= \begin{cases}1_{\lambda-\alpha_{i}} f_{i} & \text { if } \lambda-\alpha_{i} \in \Lambda(n, d), \\
0 & \text { otherwise }\end{cases} \\
& 1_{\lambda} e_{i}= \begin{cases}e_{i} 1_{\lambda-\alpha_{i}} & \text { if } \lambda-\alpha_{i} \in \Lambda(n, d), \\
0 & \text { otherwise },\end{cases} \\
& 1_{\lambda} f_{i}= \begin{cases}f_{i} 1_{\lambda+\alpha_{i}} & \text { if } \lambda+\alpha_{i} \in \Lambda(n, d), \\
0 & \text { otherwise }\end{cases} \\
& e_{i} f_{j}-f_{j} e_{i}=\delta_{i j} \sum_{\lambda \in \Lambda(n, d)}\left(\lambda_{j}-\lambda_{j+1}\right) 1_{\lambda},
\end{aligned}
$$

along with the Serre relations ( $(\underline{\mathrm{R} 4})$, (5), for $i, j \in\{1, \ldots, n-1\}$.

\section{MAin Results: QUANTIZEd CASE}

Our main results in the quantized case are similar in form to those in the classical case. The first result describes a presentation by generators and relations of the quantized Schur algebra over the rational function field $\mathbb{Q}(v)$. This presentation is compatible with the usual presentation (see section 3) of $\mathbf{U}=\mathbf{U}_{v}\left(\mathfrak{g l}_{n}\right)$.

Theorem 1'. Over $\mathbb{Q}(v)$, the $q$-Schur algebra $\mathbf{S}(n, d)$ is isomorphic with the associative algebra (with 1 ) on the same generators as for $\mathbf{U}$ subject to the same relations Q1 - Q5 as for $\mathbf{U}$, together with the additional relations:

$$
\begin{aligned}
& K_{1} K_{2} \cdots K_{n}=v^{d}, \\
& \left(K_{j}-1\right)\left(K_{j}-v\right)\left(K_{j}-v^{2}\right) \cdots\left(K_{j}-v^{d}\right)=0 \quad(j=1, \ldots, n) .
\end{aligned}
$$

The next result gives a basis for the $q$-Schur algebra which is the analogue of the Poincare-Birkhoff-Witt (PBW) type basis of $\mathbf{U}$, given in Lusztig [9, Prop. 1.13]. 
Theorem 2'. The algebra $\mathbf{S}(n, d)$ has a "truncated $P B W$ type" basis which can be described as follows. Fix an integer $k_{0}$ with $1 \leq k_{0} \leq n$ and set

$$
G^{\prime}=\left\{E_{\alpha}, F_{\alpha} \mid \alpha \in \Phi^{+}\right\} \cup\left\{K_{k} \mid k \in\{1, \ldots, n\}-\left\{k_{0}\right\}\right\}
$$

and fix some ordering for this set. Then the set of all monomials in $G^{\prime}$ (with specified order) of total degree at most $d$ is a basis for $\mathbf{S}(n, d)$.

Note that setting $v=1$ in the basis of Theorem 2 does not yield the basis of Theorem 2 since $K_{i}$ acts as the identity when $v=1$.

Our next result constructs the integral $q$-Schur algebra $\mathbf{S}_{\mathcal{A}}(n, d)$ in terms of the generators given above. For $B$ in $\mathbb{N}^{n}$, we write

$$
K_{B}=\prod_{j=1}^{n}\left[\begin{array}{c}
K_{j} \\
b_{j}
\end{array}\right]
$$

where for indeterminates $X, X^{-1}$ satisfying $X X^{-1}=X^{-1} X=1$ and any $t \in \mathbb{N}$ we formally set

$$
\left[\begin{array}{c}
X \\
t
\end{array}\right]:=\prod_{s=1}^{t} \frac{X v^{-s+1}-X^{-1} v^{s-1}}{v^{s}-v^{-s}}
$$

an expression that makes sense if $X$ is replaced by any invertible element of a $\mathbb{Q}(v)$-algebra.

Given $\lambda \in \Lambda(n, d)$ we set (when we are in the quantum case) $1_{\lambda}:=K_{\lambda}$. It turns out that, just as in the classical case, the collection $\left\{1_{\lambda}\right\}$ as $\lambda$ varies over $\Lambda(n, d)$ forms a set of pairwise orthogonal idempotents in $\mathbf{S}_{\mathcal{A}}(n, d)$ which sum to the identity element.

Let $[m]$ denote the quantum integer $[m]:=\left(v^{m}-v^{-m}\right) /\left(v-v^{-1}\right)$ and set $[m] !:=$ $[m][m-1] \cdots[1]$. Then the $q$-analogues of the divided powers of root vectors are defined to be $E_{\alpha}^{(m)}:=E_{\alpha} /[m] !$ and $F_{\alpha}^{(m)}:=F_{\alpha} /[m]$ !. Any product in $\mathbf{U}$ of elements of the form

$$
E_{\alpha}^{(m)}, \quad F_{\alpha}^{(m)}, \quad K_{j}^{ \pm 1}, \quad\left[\begin{array}{c}
K_{j} \\
m
\end{array}\right] \quad(m \in \mathbb{N}, \alpha \in \Phi, j \in\{1, \ldots, n\}),
$$

taken in any order, will be called a Kostant monomial. As before, the set of Kostant monomials is multiplicatively closed, and spans $\mathbf{U}_{\mathcal{A}}$. The definition of content $\chi$ of a Kostant monomial is obtained similarly, by setting

$$
\chi\left(E_{\alpha}^{(m)}\right)=\chi\left(F_{\alpha}^{(m)}\right):=m \varepsilon_{\max (i, j)}, \quad \chi\left(K_{l}^{ \pm 1}\right)=\chi\left(\left[\begin{array}{c}
K_{l} \\
m
\end{array}\right]\right):=0
$$

where $\alpha=\varepsilon_{i}-\varepsilon_{j} \in \Phi^{+}$, and by declaring that $\chi(X Y)=\chi(X)+\chi(Y)$ whenever $X, Y$ are Kostant monomials. For $A, C \in \mathbb{N}^{\Phi^{+}}$we write

$$
E_{A}=\prod_{\alpha \in \Phi^{+}} E_{\alpha}^{\left(a_{\alpha}\right)}, \quad F_{C}=\prod_{\alpha \in \Phi^{+}} F_{\alpha}^{\left(c_{\alpha}\right)}
$$

where the products in $E_{A}$ and $F_{C}$ are taken relative to any two specified orderings on $\Phi^{+}$.

Theorem $3^{\prime}$. The integral $q$-Schur algebra $\mathbf{S}_{\mathcal{A}}(n, d)$ is the subring of $\mathbf{S}(n, d)$ generated by all quantum divided powers $E_{j}^{(m)}, F_{j}^{(m)}(j \in\{1, \ldots, n-1\}, m \in \mathbb{N})$, along 
with the elements $\left[\begin{array}{c}K_{j} \\ m\end{array}\right](j \in\{1, \ldots, n\}, m \in \mathbb{N})$. Moreover, this algebra has a basis over $\mathcal{A}$ consisting of all

$$
E_{A} 1_{\lambda} F_{C} \quad\left(\chi\left(e_{A} f_{C}\right) \preceq \lambda\right)
$$

and another such basis consisting of all

$$
F_{A} 1_{\lambda} E_{C} \quad\left(\chi\left(f_{A} e_{C}\right) \preceq \lambda\right)
$$

where $A, C \in \mathbb{N}^{\Phi^{+}}, \lambda \in \Lambda(n, d)$, and where $\preceq$ denotes the componentwise partial ordering on $\mathbb{N}^{n}$.

Unlike the truncated PBW basis, the bases of Theorem [3] do specialize when $v=1$ to their classical analogues given in Theorem 3 ,

Finally, we have another presentation of the $q$-Schur algebra by generators and relations. This presentation has the advantage that by setting $v=1$, we recover the classical version given in Theorem 4. The relations of the presentation are similar to relations that hold for Lusztig's modified form $\dot{\mathbf{U}}$ of $\mathbf{U}$. (See [9, Chap. 23].)

Theorem 4'. For each $i \in\{1, \ldots, n-1\}$ write $\alpha_{i}:=\varepsilon_{i}-\varepsilon_{i+1}$. The algebra $\mathbf{S}(n, d)$ is the associative algebra (with 1 ) given by generators $1_{\lambda}(\lambda \in \Lambda(n, d)), E_{i}$, $F_{i}(i \in\{1, \ldots, n-1\})$ subject to the relations

$$
\begin{aligned}
& 1_{\lambda} 1_{\mu}=\delta_{\lambda, \mu} 1_{\lambda} \quad(\lambda, \mu \in \Lambda(n, d)), \\
& \sum_{\lambda \in \Lambda(n, d)} 1_{\lambda}=1 \\
& E_{i} 1_{\lambda}= \begin{cases}1_{\lambda+\alpha_{i}} E_{i} & \text { if } \lambda+\alpha_{i} \in \Lambda(n, d), \\
0 & \text { otherwise, }\end{cases} \\
& F_{i} 1_{\lambda}= \begin{cases}1_{\lambda-\alpha_{i}} F_{i} & \text { if } \lambda-\alpha_{i} \in \Lambda(n, d), \\
0 & \text { otherwise }\end{cases} \\
& 1_{\lambda} E_{i}= \begin{cases}E_{i} 1_{\lambda-\alpha_{i}} & \text { if } \lambda-\alpha_{i} \in \Lambda(n, d), \\
0 & \text { otherwise, }\end{cases} \\
& 1_{\lambda} F_{i}= \begin{cases}F_{i} 1_{\lambda+\alpha_{i}} & \text { if } \lambda+\alpha_{i} \in \Lambda(n, d), \\
0 & \text { otherwise }\end{cases} \\
& E_{i} F_{j}-F_{j} E_{i}=\delta_{i j} \sum_{\lambda \in \Lambda(n, d)}\left[\lambda_{j}-\lambda_{j+1}\right] 1_{\lambda},
\end{aligned}
$$

along with the $q$-Serre relations QQ4, Q5, for $i, j \in\{1, \ldots, n-1\}$.

\section{Other Results}

6.1. Triangular decomposition. One can define the plus, minus, and zero parts of Schur algebras in terms of the generators, as follows. (These subalgebras have been studied before.) The plus part $S^{+}(n, d)$ (resp., the minus part $S^{-}(n, d)$ ) is the subalgebra of $S(n, d)$ generated by all $x_{\alpha}, \alpha \in \Phi^{+}$(resp., $\alpha \in \Phi^{-}$). The zero part $S^{0}(n, d)$ is the subalgebra generated by all $H_{j}, j=1, \ldots, n$. We also have the Borel Schur algebras $S^{\geqslant 0}(n, d)$ (resp., $S^{\leqslant 0}(n, d)$ ), the subalgebra generated by $S^{+}(n, d)$ (resp., $\left.S^{-}(n, d)\right)$ together with $S^{0}(n, d)$. 
The appellations $S_{\mathbb{Z}}^{+}(n, d), S_{\mathbb{Z}}^{-}(n, d), S_{\mathbb{Z}}^{0}(n, d), S_{\mathbb{Z}}^{\geqslant 0}(n, d), S_{\mathbb{Z}}^{\leqslant 0}(n, d)$ will denote the intersection of the appropriate algebra from above with the integral form $S_{\mathbb{Z}}(n, d)$.

The algebra $S=S(n, d)$ has a triangular decomposition $S=S^{+} S^{0} S^{-}$. We show that in this decomposition one can permute the three factors in any order. Moreover, the same result holds over $\mathbb{Z}$.

The zero part $S_{\mathbb{Z}}^{0}(n, d)$ is the algebra generated by all $\left(\begin{array}{c}H_{j} \\ m\end{array}\right)$ for $j=1, \ldots, n$ and $m \in \mathbb{N}$. We give in [4] a presentation of $S^{0}(n, d)$ by generators and relations. In particular, we prove that $H_{B}=0$ whenever $|B|>d\left(B \in \mathbb{N}^{n}\right)$ and that the set of (pairwise orthogonal) idempotents $1_{\lambda}, \lambda \in \Lambda(n, d)$, is a $\mathbb{Z}$-basis of $S_{\mathbb{Z}}^{0}(n, d)$.

$S_{\mathbb{Z}}^{+}(n, d)$ (resp., $\left.S_{\mathbb{Z}}^{-}(n, d)\right)$ is the algebra generated by all divided powers $x_{\alpha}^{(m)}$ for $\alpha \in \Phi^{+}$(resp., $\alpha \in \Phi^{-}$) and $m \in \mathbb{N}$. It is an easy consequence of the commutation formulas (S2) that each generator $x_{\alpha}$ is nilpotent of index $d+1$; see 4 for details. Moreover, from our main results we see easily that the set of all $e_{A}$ (resp., $f_{A}$ ) such that $|A| \leq d$ is a $\mathbb{Z}$-basis for the algebra $S_{\mathbb{Z}}^{+}(n, d)$ (resp., $\left.S_{\mathbb{Z}}^{-}(n, d)\right)$.

It also follows immediately from our results that the set of all $e_{A} 1_{\lambda}$ (resp., $1_{\lambda} f_{A}$ ) satisfying $\chi\left(e_{A}\right) \preceq \lambda$ is an integral basis for the Borel Schur algebra $S_{\mathbb{Z}}^{\geqslant 0}(n, d)$ (resp., $\left.S_{\mathbb{Z}}^{\leqslant 0}(n, d)\right)$.

Similar statements to the above hold in the quantum case. In particular, as a corollary of the commutation formulas (S2] ) one can give a simple proof of [7] Prop. $2.3]$.

6.2. Explicit reduction formulas. Fix a positive root $\alpha$ and write $\alpha=\varepsilon_{i}-\varepsilon_{j}$ for $i<j$.

Then from [2] we have the following reduction formulas in $S_{\mathbb{Z}}(n, d)$, for any $a, b, c \in \mathbb{N}:$

$$
\begin{aligned}
& f_{\alpha}^{(a)}\left(\begin{array}{c}
H_{j} \\
b
\end{array}\right) e_{\alpha}^{(c)}=\sum_{k=s}^{\min (a, c)}(-1)^{k-s}\left(\begin{array}{c}
k-1 \\
s-1
\end{array}\right)\left(\begin{array}{c}
b+k \\
k
\end{array}\right) f_{\alpha}^{(a-k)}\left(\begin{array}{c}
H_{j} \\
b+k
\end{array}\right) e_{\alpha}^{(c-k)}, \\
& e_{\alpha}^{(a)}\left(\begin{array}{c}
H_{i} \\
b
\end{array}\right) f_{\alpha}^{(c)}=\sum_{k=s}^{\min (a, c)}(-1)^{k-s}\left(\begin{array}{c}
k-1 \\
s-1
\end{array}\right)\left(\begin{array}{c}
b+k \\
k
\end{array}\right) e_{\alpha}^{(a-k)}\left(\begin{array}{c}
H_{i} \\
b+k
\end{array}\right) f_{\alpha}^{(c-k)},
\end{aligned}
$$

where $s=a+b+c-d$ and $s \geq 1$.

We do not have a $q$-analogue of these formulas.

The results of [3] give another type of reduction formula for $\mathbf{S}_{\mathcal{A}}(n, d)$. If $b_{1}, b_{2} \in \mathbb{N}$ satisfy $b_{1}+b_{2}=d$, set $\lambda:=b_{1} \varepsilon_{i}+b_{2} \varepsilon_{j} \in \Lambda(n, d)$. Then for all $s \geq 1$ we have:

$$
\begin{aligned}
& E_{\alpha}^{(a)} 1_{\lambda} F_{\alpha}^{(c)}=\sum_{k=s}^{\min (a, c)}(-1)^{k-s}\left[\begin{array}{c}
k-1 \\
s-1
\end{array}\right]\left[\begin{array}{c}
b_{1}+k \\
k
\end{array}\right] E_{\alpha}^{(a-k)} 1_{\lambda+k \alpha} F_{\alpha}^{(c-k)}, \\
& F_{\alpha}^{(a)} 1_{\lambda} E_{\alpha}^{(c)}=\sum_{k=s}^{\min (a, c)}(-1)^{k-s}\left[\begin{array}{c}
k-1 \\
s-1
\end{array}\right]\left[\begin{array}{c}
b_{2}+k \\
k
\end{array}\right] F_{\alpha}^{(a-k)} 1_{\lambda-k \alpha} E_{\alpha}^{(c-k)},
\end{aligned}
$$

where $s=a+b_{1}+c-d$ in (3) and $s=a+b_{2}+c-d$ in (4).

The classical analogues of formulas (3) and (4) hold in $S(n, d)$.

6.3. Another presentation (for $n=2$ ). We have the following result from [2], which presents $S(2, d)$ as a quotient of $U\left(\mathfrak{s l}_{2}\right)$. 
Theorem 5. Over $\mathbb{Q}$, the Schur algebra $S(2, d)$ is isomorphic with the associative algebra (with 1) generated by $e, f, h$ subject to the relations:

$$
\begin{aligned}
& h e-e h=2 e ; \quad \text { ef }-f e=h ; \quad h f-f h=-2 f ; \\
& (h+d)(h+d-2) \cdots(h-d+2)(h-d)=0 .
\end{aligned}
$$

Moreover, this algebra has a "truncated $P B W$ " basis over $\mathbb{Q}$ consisting of all $f^{a} h^{b} e^{c}$ such that $a+b+c \leq d$.

Note that if we eliminate the last relation, we have the usual presentation of $U\left(\mathfrak{s} \mathfrak{l}_{2}\right)$ over $\mathbb{Q}$. The last relation is the minimal polynomial of $h$ in the representation on tensor space. The problem of presenting $S(n, d)$ as a quotient of $U\left(\mathfrak{s l}_{n}\right)$ seems to be more difficult for $n>2$.

We also have from [3] the following $q$-version of the above, which presents the $q$-Schur algebra $\mathbf{S}(2, d)$ as a quotient of the quantized enveloping algebra $\mathbf{U}\left(\mathfrak{s l}_{2}\right)$.

Theorem 5'. Over $\mathbb{Q}(v)$, the quantum Schur algebra $\mathbf{S}(2, d)$ is isomorphic to the algebra generated by $E, F, K^{ \pm 1}$ subject to the relations:

$$
\begin{aligned}
& K K^{-1}=K^{-1} K=1, \\
& K E K^{-1}=v^{2} E, \quad K F K^{-1}=v^{-2} F, \\
& E F-F E=\frac{K-K^{-1}}{v-v^{-1}}, \\
& \left(K-v^{d}\right)\left(K-v^{d-2}\right) \cdots\left(K-v^{-d+2}\right)\left(K-v^{-d}\right)=0 .
\end{aligned}
$$

6.4. Hecke algebras. Suppose that $n \geq d$. Let $\omega=\left(1^{d}\right)$. Then the subalgebra $1_{\omega} \mathbf{S}(n, d) 1_{\omega}$ is isomorphic with the Hecke algebra $\mathbf{H}\left(\Sigma_{d}\right)$. The nonzero elements of the basis (B1') of the form $1_{\omega} E_{A} F_{C} 1_{\omega}$ form a basis of the Hecke algebra; similarly for elements of the basis $\left(\mathrm{B} 2^{\prime}\right)$ of the form $1_{\omega} F_{A} E_{C} 1_{\omega}$.

Moreover, taking $d=n$, we can see that $\mathbf{H}=\mathbf{H}\left(\Sigma_{n}\right)$ is generated by the elements $1_{\omega} E_{i} F_{i} 1_{\omega}(1 \leq i \leq n-1)$. Alternatively, $\mathbf{H}$ is generated by the $1_{\omega} F_{i} E_{i} 1_{\omega}$ $(1 \leq i \leq n-1)$. One can easily describe the relations on these generators, thus obtaining a presentation of $\mathbf{H}$ which is closely related to one in 10 .

\section{REFERENCES}

1. A.A. Beilinson, G. Lusztig, and R. MacPherson, A geometric setting for the quantum deformation of $\mathrm{GL}_{n}$, Duke Math. J. 61 (1990), 655-677. MR 91m:17012

2. S. Doty and A. Giaquinto, Presenting Schur algebras as quotients of the universal enveloping algebra of $\mathfrak{g l}_{2}$, Algebras and Representation Theory, to appear.

3. S. Doty and A. Giaquinto, Presenting quantum Schur algebras as quotients of the quantized enveloping algebra of $\mathfrak{g l}_{2}$, preprint, Loyola University Chicago, December 2000.

4. S. Doty and A. Giaquinto, Presenting Schur algebras, preprint, Loyola University Chicago, April 2001.

5. Jie Du, A note on quantized Weyl reciprocity at roots of unity, Algebra Colloq. 2 (1995), 363-372. MR 96m:17024

6. J. A. Green, Polynomial Representations of $\mathrm{GL}_{n}$ (Lecture Notes in Math. 830), SpringerVerlag, New York 1980. MR 83j:20003

7. R. Green, $q$-Schur algebras as quotients of quantized enveloping algebras, J. Algebra 185 (1996), 660-687. MR 97k:17016

8. M. Jimbo, A $q$-analogue of $U(\mathfrak{g l}(N+1))$, Hecke algebra, and the Yang-Baxter equation, Letters Math. Physics 11 (1986), 247-252. MR 87k:17011 
9. G. Lusztig, Introduction to Quantum Groups, Birkhäuser Boston 1993. MR 94m:17016

10. H. Wenzl, Hecke algebras of type $A_{n}$ and subfactors, Invent. Math. 92 (1988), 349-383. MR 90b:46118

Department of Mathematics, Loyola University, Chicago, IL 60626

E-mail address: doty@math.luc.edu

Department of Mathematics, Loyola University, Chicago, IL 60626

E-mail address: tonyg@math.luc.edu 